Supporting Information (SI) for

\title{
A Dynamic Interface Based on Segregation of an Amphiphilic Hyperbranched Polymer Containing Fluoroalkyl and Oligo(ethylene oxide) Moieties
}

Yukari Oda, ${ }^{\dagger, \| \#}$ Manabu Inutsuka, ${ }^{\dagger, \#, \|}$ Ryo Awane, ${ }^{\dagger}$ Masayasu Totani, ${ }^{\dagger}$ Norifumi L. Yamada, ${ }^{\ddagger}$ Masayuki Haraguchi ${ }^{\S}$ Masaaki Ozawa, ${ }^{\S}$ Hisao Matsuno, ${ }^{\dagger, \uparrow, \%}$ and Keiji Tanaka*,†,,$\%$

${ }^{\dagger}$ Department of Applied Chemistry, Kyushu University, Fukuoka 819-0395, Japan

${ }^{\ddagger}$ Neutron Science Division, Institute of Materials Structure Science, High Energy Acceleration Research Organization, Ibaraki 319-1106, Japan

${ }^{\S}$ Materials Research Laboratories, Nissan Chemical Corporation, Chiba 274-0052, Japan

"Center for Polymer Interface and Molecular Adhesion Science, Kyushu University, Fukuoka 819-0395, Japan

${ }^{\%}$ International Institute for Carbon-Neutral Energy Research (WPI-I2CNER), Kyushu University, Fukuoka 819-0395, Japan

"Both authors contributed equally to this work.

Corresponding author: *k-tanaka@ cstf.kyushu-u.ac.jp 


\section{Synthesis of hyperbranched polymers.}

A hyperbranched polymer (HBP) containing both fluoroalkyl $\left(\mathrm{R}_{\mathrm{f}}\right.$ : $\left.-\mathrm{C}_{6} \mathrm{~F}_{13}\right)$ pendants and carboxy end groups, $\mathrm{HBP}-\mathrm{COOH}$, was prepared by initiator-fragment incorporation radical polymerization, ${ }^{\mathrm{S} 1}$ as previously reported. ${ }^{\mathrm{S} 2}$ Scheme S1 shows a synthetic route of HBP- $n \mathrm{EO}$ by condensation reaction [HBP- $n$ EO: HBP containing both $\mathrm{R}_{\mathrm{f}}$ pendants and oligo(ethylene oxide) with various repeating units $(n)$ $(n \mathrm{EO})$ at chain ends].

Scheme S1. Synthetic scheme of HBP- $n$ EO based on condensation reaction of HBP-COOH with oligo(ethylene glycol) monomethyl ether (repeating unit, $n=6,12$, and 45). DIC: $N, N$ '-diidopropylcarbodiimide, DMAP: 4-dimethylaminopyridine, THF: tetrahydrofuran.

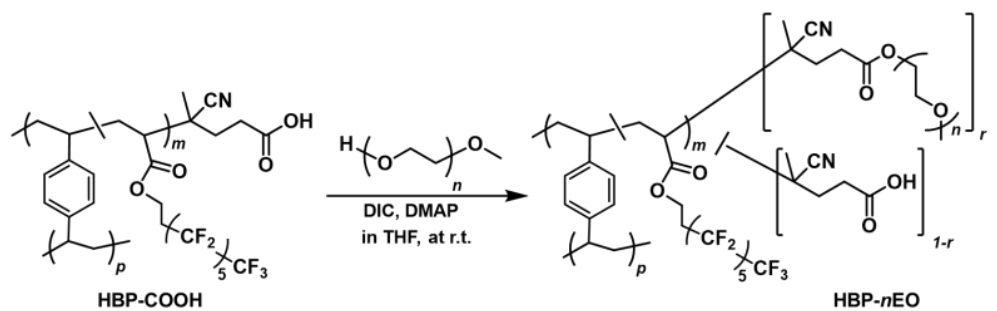

Figure $\mathrm{S} 1$ shows ${ }^{1} \mathrm{H}$ nuclear magnetic resonance (NMR) spectra for $\mathrm{HBP}-n \mathrm{EO}$ in acetone- $d_{6}$. End group conversion was calculated comparing the integral area between signals for aromatic protons $(a)$ and those for methylene protons adjacent to ester groups $(f)$ or oligo(ethylene oxide) units $(k)$.
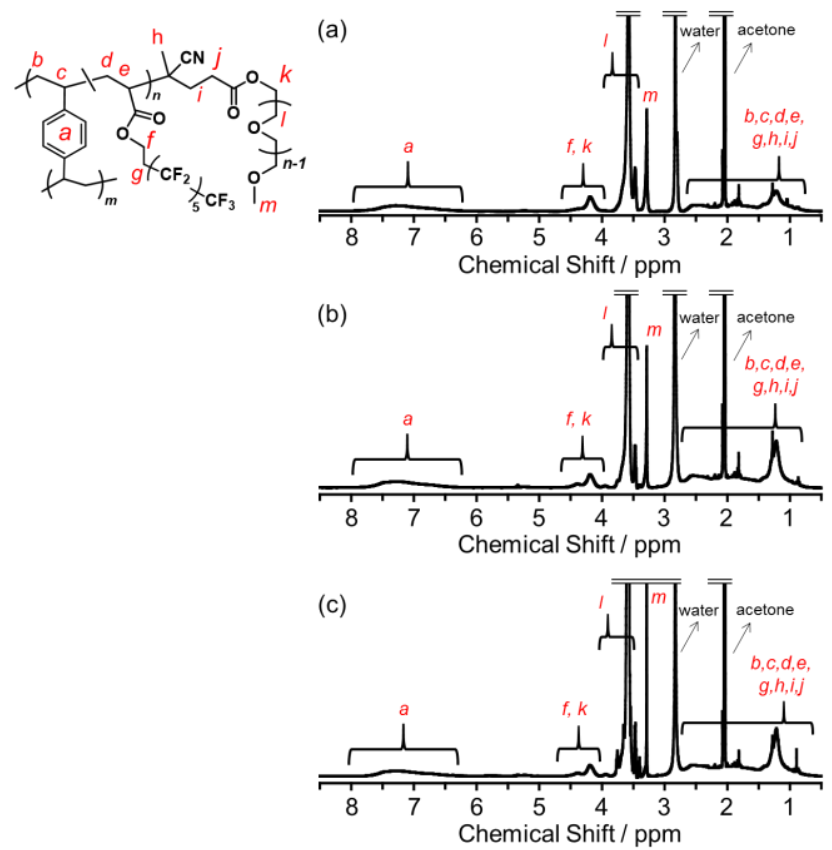

Figure S1. $\quad{ }^{1} \mathrm{H}$ NMR spectra for HBP- $n$ EO in acetone- $d_{6}$; (a) $n=6$, (b) $n=12$, and (c) $n=45$. 


\section{Surface and interfacial characterization.}

2-1. Surface morphology. Root-mean-square roughness of polymer films was examined by atomic force microscopy (AFM) with a dynamic force mode in air and water at room temperature. Figure S2 shows AFM topographic images for the HBP- $n$ EO/PMMA blend films in air; (a) $n=6$, (b) $n=$ 12 , and (c) $n=45$. Even though the film was immersed in water, the appearance of the film surface remained unchanged.
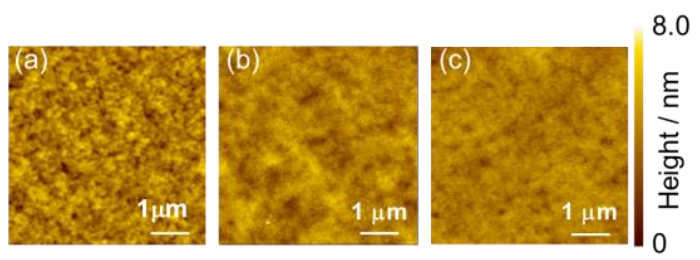

Figure S2. AFM topographic images for HBP- $n$ EO/PMMA films in air; (a) $n=6$, (b) $n=12$, and (c) $n=45$.

2-2. Chemical composition. Chemical composition at the surface in the blend films was examined by angular dependent X-ray photoelectron spectroscopy (AD-XPS) measurement. Figure S3 shows typical XPS $F_{1 S}$ and $\mathrm{C}_{1 \mathrm{~S}}$ core-level spectra for the HBP- $n \mathrm{EO} / \mathrm{PMMA}$ films collected at an emission angle of photoelectrons $\left(\theta_{\mathrm{e}}\right)$ of $45^{\circ}$. The $\mathrm{F}_{1 \mathrm{~s}}$ peak was observed around $687-688 \mathrm{eV}$. The $\mathrm{C}_{1 \mathrm{~s}}$ peaks corresponding neutral, ether (and cyano), carbonyl, and fluoro $\left(-\mathrm{CF}_{2}\right)$ carbons were observed at 285.0, 286.5, 289.0, and $291.5 \mathrm{eV}$, respectively. Similar spectra were obtained for all blend films.
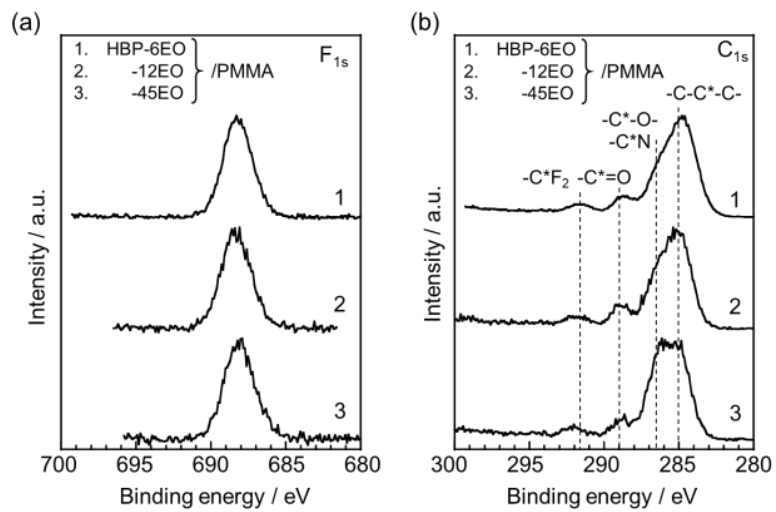

Figure S3. Typical XPS (a) $\mathrm{F}_{1 \mathrm{~S}}$ and (b) $\mathrm{C}_{1 \mathrm{~s}}$ core-level spectra for HBP- $n$ EO/PMMA films collected at $\theta_{\mathrm{e}}$ of $45^{\circ}$.

The fraction of the area occupied by $\mathrm{R}_{\mathrm{f}}$ chains $(f)$ was calculated by the following equation:

$$
\left(n_{\mathrm{F}} / n_{\mathrm{C}}\right)=\left(n_{\mathrm{F}, 1} \bullet f\right) /\left\{n_{\mathrm{C}, 1} \bullet f+n_{\mathrm{C}, 2} \bullet(1-f)\right\}
$$

where $n_{\mathrm{i}, 1}$ and $n_{\mathrm{i}, 2}$ are number density of $i$ atom in $\mathrm{R}_{\mathrm{f}}$ chains and rest part of HBP- $n \mathrm{EO}$. Since the $\left(n_{\mathrm{F}} / n_{\mathrm{C}}\right)$ value in the segregation layer was 0.76 for all $n$, the $f$ values were calculated to be $0.54,0.52$, and 
0.48 for $n=6,12$, and 45 , respectively. The number of $R_{\mathrm{f}}$ chains per unit area $\left(N_{\mathrm{Rf}}\right)$ was obtained as follows:

$$
N_{\mathrm{Rf}}=(d \bullet f) / V_{\mathrm{Rf}}
$$

where $d$ and $V_{\mathrm{Rf}}$ are thickness of the $\mathrm{R}_{\mathrm{f}}$ segregation layer and volume of a single $\mathrm{R}_{\mathrm{f}}$ chain, respectively. Assuming that $d$ and $f$ were 0.58 and 0.5 for all $n$, the $N_{\mathrm{Rf}}$ value was calculated to be 0.9 chains* $\mathrm{nm}^{-2}$.

2-3. Depth profile in air. Figure $S 4$ shows a schematic illustration of a four-layer model, in which $t_{\mathrm{j}}$ and $\sigma_{\mathrm{j}}$ are the thickness and interfacial roughness of each layer, respectively, to fit experimental neutron reflectivity (NR) curves for the blend films in air. The subscript $j$ refers to the $j$ th layer from the air side. Figure S5 represents NR curves and model $(b / V)$ profiles for the HBP- $n$ EO/dPMMA films in air. Here, $q$ is scattering vector $[=(4 \pi / \lambda) \sin \theta$, where $\lambda$ and $\theta$ are neutron wavelength and incident angle, respectively]. Table S1 summarizes parameters used to fit experimental reflectivity curves acquired in air.

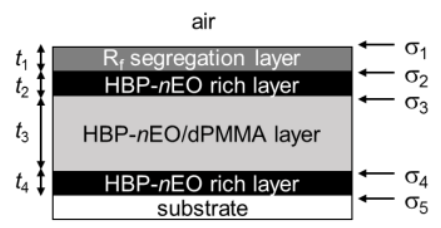

Figure S4. A schematic illustration of a model to fit experimental reflectivity curves acquired in air.
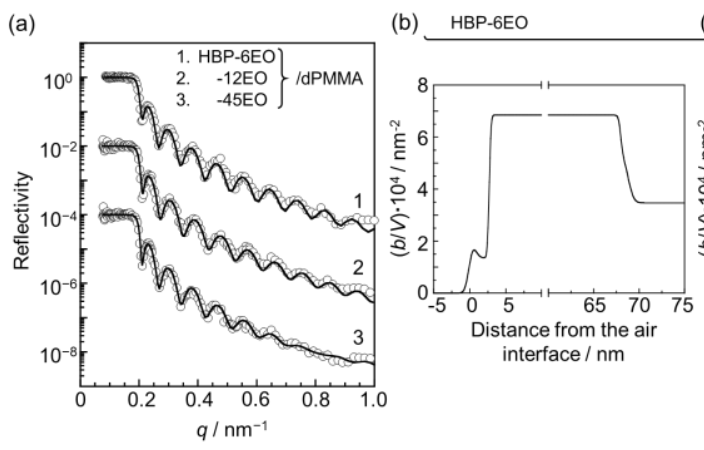

(c) $-12 \mathrm{EO}$

(d) $-45 \mathrm{EO}$
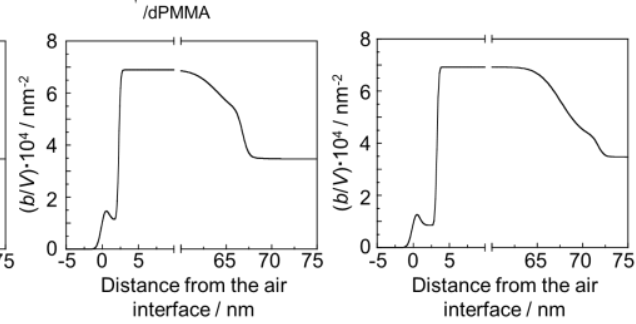

Figure S5. (a) NR profiles for HBP- $n$ EO/dPMMA in air. Open symbols demote experimental data and solid curves are calculated reflectivity based on the model $(b / V)$ profiles shown in panels (b), (c), and (d) for HBP-6EO, -12EO, and -45EO, respectively.

Table S1. Parameters used to fit experimental reflectivity curves acquired in air, as shown in Figure S5(a). The subscript $i$ refers to the $i$ th layer from the air side.

\begin{tabular}{|c|c|c|c|c|c|c|c|c|c|c|c|c|c|}
\hline \multirow{2}{*}{ Sample } & \multicolumn{4}{|c|}{$t_{\mathrm{i}} / \mathrm{nm}$} & \multicolumn{4}{|c|}{$(b / V)_{\mathrm{i}} \cdot 10^{4} / \mathrm{nm}^{-2}$} & \multicolumn{5}{|c|}{$\sigma_{\mathrm{i}} / \mathrm{nm}$} \\
\hline & $t_{1}$ & $t_{2}$ & $t_{3}$ & $t_{4}$ & $(b / V)_{1}$ & $(b / V)_{2}$ & $(b / V)_{3}$ & $(b / V)_{4}$ & $\sigma_{1}$ & $\sigma_{2}$ & $\sigma_{3}$ & $\sigma_{4}$ & $\sigma_{5}$ \\
\hline HBP-6EO/dPMMA & 0.58 & 2.1 & 65.3 & 0.8 & 2.60 & 1.36 & 6.85 & 5.49 & 0.5 & 0.5 & 0.2 & 0.2 & 0.5 \\
\hline HBP-12EO/dPMMA & 0.58 & 1.7 & 61.9 & 2.7 & 2.40 & 1.12 & 6.89 & 5.11 & 0.5 & 0.5 & 0.2 & 2.0 & 0.5 \\
\hline HBP-45EO/dPMMA & 0.58 & 2.7 & 64.4 & 4.1 & 2.20 & 0.855 & 6.92 & 4.20 & 0.5 & 0.5 & 0.2 & 2.0 & 0.5 \\
\hline
\end{tabular}


2-4. Interfacial stability. Contact angle $(\phi)$ of an air bubble with a volume of $2.0 \mu \mathrm{L}$ against the polymer films in Milli-Q water was recorded as a function of immersion time $(t)$. Figure S6 shows $t$ dependence of $\phi$ for the HBP-12EO/PMMA blend film in water at room temperature and $310 \mathrm{~K}$. The data at room temperature was imported from Figure 6. Since the absolute $\phi$ values for the two temperatures at a given time could be not the same, the data sets were fitted by the following equation;

$$
\phi(t)=\left(\phi_{\text {ini }}-\phi_{\text {ter }}\right) \cdot \exp (-t / \tau)+\phi_{\text {ter }}
$$

where $\phi_{\mathrm{ini}}, \phi_{\mathrm{ter}}$, and $\tau$ are initial and terminal $\phi$ values and the time constant of the $\phi$ change, respectively. The contact angle of an air bubble on the HBP-12EO/PMMA film in water was almost constant for the immersion time longer than 3 hours at $310 \mathrm{~K}$. Thus, it is apparent that the surface of the HBP-12EO/PMMA film was stable even at $310 \mathrm{~K}$.

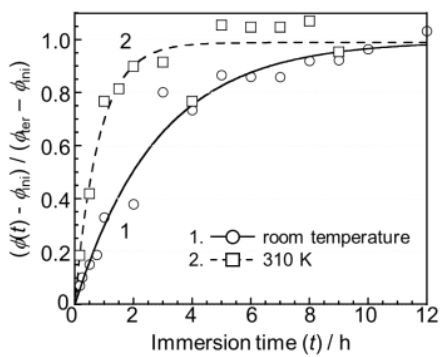

Figure S6. Immersion time $(t)$ dependence of $\phi$ for HBP-12EO/PMMA in water at room temperature and $310 \mathrm{~K}$.

2-5. Depth profiles in water. Figure $S 7$ shows a schematic illustration of a four-layer model to fit experimental NR curves in water. Figures S8-S10 represent NR curves and model $(b / V)$ profiles for HBP- $n \mathrm{EO} /(\mathrm{d}) \mathrm{PMMA}$ film in $\mathrm{D}_{2} \mathrm{O}$. Table $\mathrm{S} 2$ summarizes parameters used to fit experimental reflectivity curves acquired in $\mathrm{D}_{2} \mathrm{O}$.

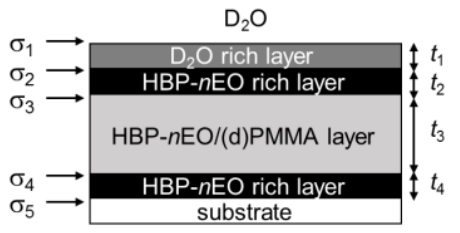

Figure S7. A schematic illustration of a model to fit experimental reflectivity curves in $\mathrm{D}_{2} \mathrm{O}$. 


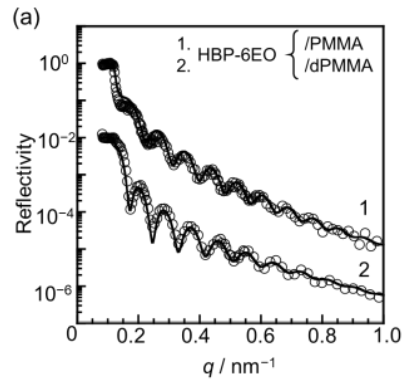

(b)

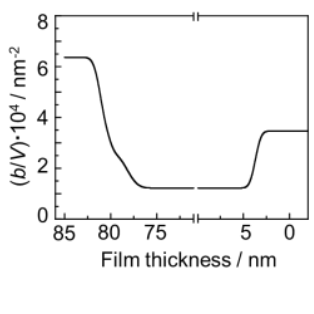

(c)

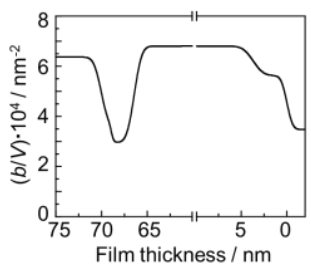

Figure S8. (a) NR profiles for HBP-6EO/PMMA and HBP-6EO/dPMMA in $\mathrm{D}_{2} \mathrm{O}$. Open symbols demote experimental data and solid curves are calculated reflectivity based on the model $(b / V)$ profiles shown in panels (b) and (c) for HBP-6EO/PMMA and HBP-6EO/dPMMA, respectively.

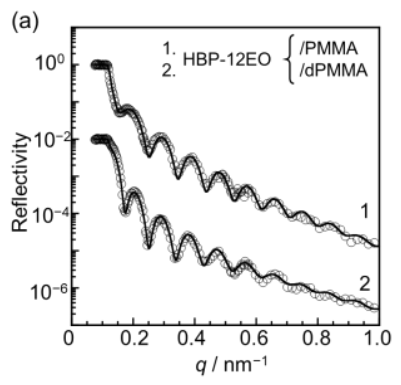

(b)

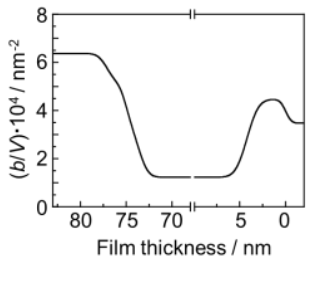

(c)

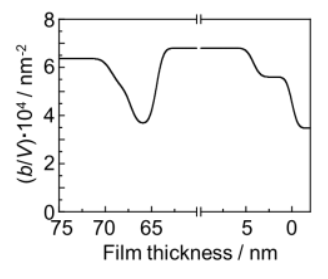

Figure S9. (a) NR profiles for HBP-12EO/PMMA and HBP-12EO/dPMMA in $\mathrm{D}_{2} \mathrm{O}$. Open symbols demote experimental data and solid curves are calculated reflectivity based on the model $(b / V)$ profiles shown in panels (b) and (c) for HBP-12EO/PMMA and HBP-12EO/dPMMA, respectively.

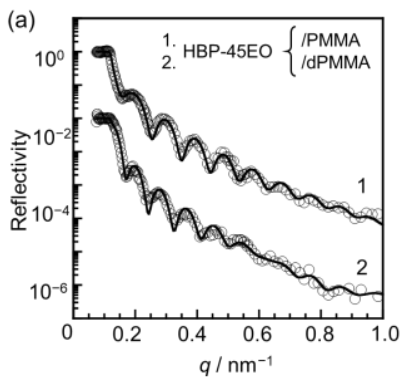

(b)

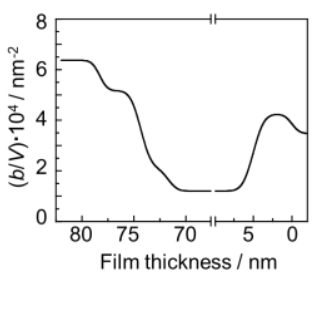

(c)

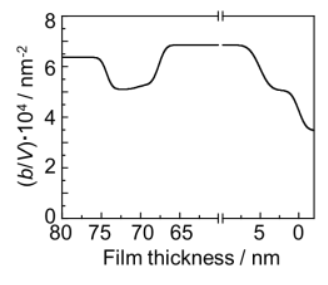

Figure S10. (a) NR profiles for HBP-45EO/PMMA and HBP-45EO/dPMMA in $\mathrm{D}_{2} \mathrm{O}$. Open symbols demote experimental data and solid curves are calculated reflectivity based on the model $(b / V)$ profiles shown in panels (b) and (c) for HBP-45EO/PMMA and HBP-45EO/dPMMA, respectively.

Table S2. Parameters used to fit experimental reflectivity curves in $\mathrm{D}_{2} \mathrm{O}$ as shown in Figures $\mathrm{S} 8(\mathrm{a}), \mathrm{S} 9$ (a), and $\mathrm{S} 10$ (a). The subscript $i$ refers to the $i$ th layer from the $\mathrm{D}_{2} \mathrm{O}$ side.

\begin{tabular}{|c|c|c|c|c|c|c|c|c|c|c|c|c|c|}
\hline \multirow{2}{*}{ Sample } & \multicolumn{4}{|c|}{$t_{\mathrm{i}} / \mathrm{nm}$} & \multicolumn{4}{|c|}{$(b / V) \mathrm{i}^{*} 10^{4} / \mathrm{nm}^{-2}$} & \multicolumn{5}{|c|}{$\sigma_{1} / \mathrm{nm}$} \\
\hline & $t_{1}$ & $t_{2}$ & $t_{3}$ & $t_{4}$ & $(b / V)_{1}$ & $(b / V)_{2}$ & $(b / V)_{3}$ & $(b / V)_{4}$ & $\sigma_{1}$ & $\sigma_{2}$ & $\sigma_{3}$ & $\sigma_{4}$ & $\sigma_{5}$ \\
\hline HBP-6EO/PMMA & 1.1 & 1.8 & 74.5 & 3.7 & 3.26 & 2.65 & 1.22 & 3.47 & 0.6 & 0.4 & 0.7 & 0.5 & 0.5 \\
\hline HBP-6EO/dPMMA & 1.1 & 2.5 & 62.8 & 3.7 & 3.36 & 2.75 & 6.80 & 5.62 & 0.6 & 0.4 & 0.7 & 0.6 & 0.5 \\
\hline HBP-12EO/PMMA & 2.0 & 2.0 & 68.8 & 3.9 & 5.13 & 3.55 & 1.22 & 4.47 & 0.8 & 0.6 & 0.6 & 0.8 & 0.5 \\
\hline HBP-12EO/dPMMA & 2.0 & 2.8 & 61.0 & 4.2 & 5.14 & 3.66 & 6.88 & 5.60 & 0.8 & 0.6 & 0.6 & 0.8 & 0.5 \\
\hline HBP-45EO/PMMA & 4.0 & 2.6 & 66.7 & 5.0 & 5.17 & 2.11 & 1.22 & 4.24 & 0.9 & 0.8 & 0.7 & 1.1 & 0.6 \\
\hline HBP-45EO/dPMMA & 4.0 & 2.7 & 62.6 & 5.1 & 5.10 & 5.29 & 6.85 & 5.06 & 0.9 & 0.8 & 0.7 & 1.1 & 0.6 \\
\hline
\end{tabular}




\section{References}

S1) Sato, T.; Sato, N.; Seno, M.; Hirano, T. Initiator-Fragment Incorporation Radical Polymerization of Divinylbenzene in the Presence of Glyoxylic Oxime Ether: Formation of Soluble Hyperbranched Polymer. J. Polym. Sci., Part A; Polym. Chem. 2003, 41, 3038-3047.

S2) Haraguchi, M.; Hideki, M.; Satoru, H.; Ozawa, M. WO2010126140, November 4, 2010. 